ANL-18/10

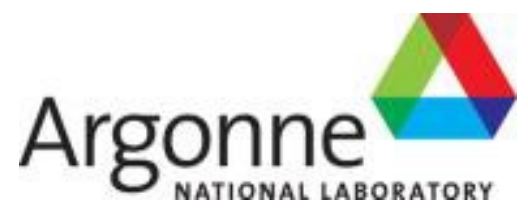

\title{
Guidance on How to Report Testing of BOP Shear Rams
}

Guidance and Clarification on How to Report Testing of Blowout Preventer (BOP) Shear Rams Used for Outer Continental Shelf (OCS) Oil and Gas Operations

\section{Energy Systems Division}




\section{Report Authors}

This report was prepared by the Global Energy Solutions (GES) Center team under the direction of Joe Braun.

\section{About Argonne National Laboratory}

Argonne is a U.S. Department of Energy laboratory managed by UChicago Argonne, LLC under contract DE-AC02-06CH11357. The Laboratory's main facility is outside Chicago, at 9700 South Cass Avenue, Argonne, Illinois 60439. For information about Argonne and its pioneering science and technology programs, see www.anl.gov.

\section{DOCUMENT AVAILABILITY}

Online Access: U.S. Department of Energy (DOE) reports produced after 1991 and a growing number of pre-1991 documents are available free via DOE's SciTech Connect

(http://www.osti.gov/scitech/).

Reports not in digital format may be purchased by the public from the National Technical Information Service (NTIS):

U.S. Department of Commerce

National Technical Information Service

5301 Shawnee Rd

Alexandria, VA22312

www.ntis.gov

Phone: (800) 553-NTIS (6847) or (703) 605-6000

Fax: (703) 605-6900

Email: orders@ntis.gov

Reports not in digital format are available to DOE and DOE contractors from the

Office of Scientific and Technical Information (OSTI):

U.S. Department of Energy

Office of Scientific and Technical Information

P.O. Box 62

Oak Ridge, TN 37831-0062

www.osti.gov

Phone: (865) 576-8401

Fax: (865) 576-5728

Email: reports@osti.gov

Disclaimer

This report was prepared as an account of work sponsored by an agency of the United States Government. Neither the United States Government nor any agency thereof, nor UChicago Argonne, LLC, nor any of their employees or officers, makes any warranty, express or implied, or assumes any legal liability or responsibility for the accuracy, completeness, or usefulness of any information, apparatus, product, or process disclosed, or represents that its use would not infringe privately owned rights. Reference herein to any specific commercial product, process, or service by trade name, trademark, manufacturer, or otherwise, does not necessarily constitute or imply its endorsement, recommendation, or favoring by the United States Government or any agency thereof. The views and opinions of document authors expressed herein do not necessarily state or reflect those of the United States Government or any agency thereof, Argonne National Laboratory, or UChicago Argonne, LLC.

This report was prepared by Argonne National Laboratory (ANL) under contract to the Department of Energy (DOE) through an interagency agreement between the Department of the Interior, Bureau of Safety and Environmental Enforcement (BSEE) and the DOE. The opinions, findings, conclusions, and recommendations expressed in the report are those of the authors and they do not necessarily reflect the views or policies of BSEE. 


\section{Guidance on How to Report Testing of BOP Shear Rams}

Guidance and Clarification on How to Report Testing of Blowout Preventer (BOP) Shear Rams Used for Outer Continental Shelf (OCS) Oil and Gas Operations

prepared by

Roy A. Lindley

Energy Systems Division, Argonne National Laboratory

Prepared for the U.S. Department of the Interior, Bureau of Safety and Environmental Enforcement

May 1, 2018 


\section{Contents}

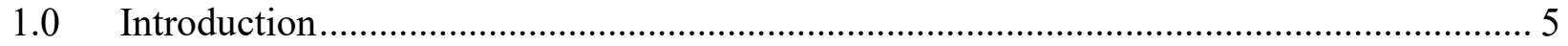

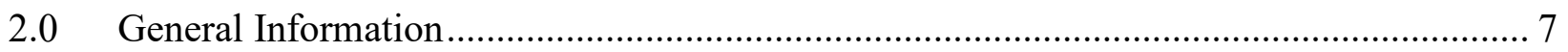

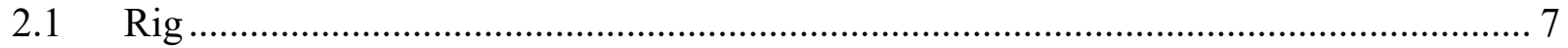

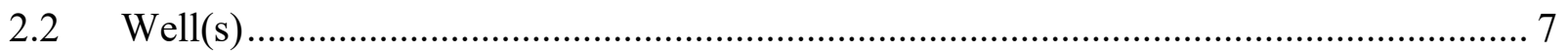

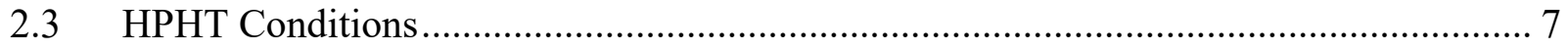

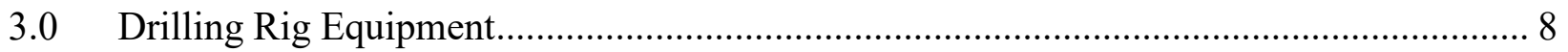

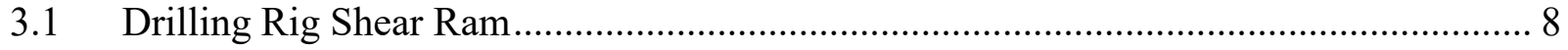

3.2 Drilling Rig Shearable Tubulars and Wireline.......................................................... 9

3.3 Drilling Rig Shear Ram Support Systems................................................................. 11

3.4 Anticipated Well Conditions Pertinent to Shear Ram................................................. 11

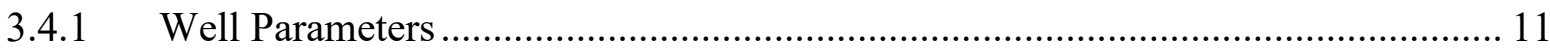

3.4.2 Maximum Anticipated Surface Pressure (MASP) ................................................. 11

3.4.3 Maximum Anticipated Well Pressures and Temperatures...................................... 12

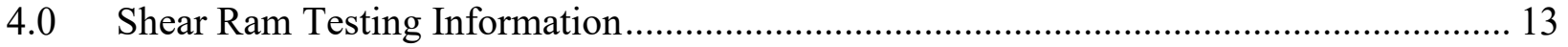

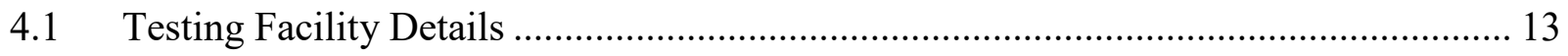

4.2 Shear Ram(s) Tested .......................................................................................... 14

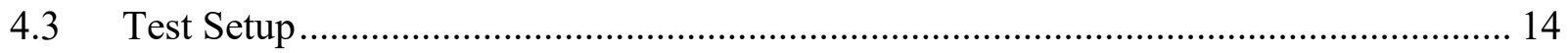

4.4 Test Procedure/Protocol Followed and Acceptance Criteria …………………............. 15

4.5 Test Specimen Selection .................................................................................. 15

4.6 Shear Ram Inspection Information ...................................................................... 16

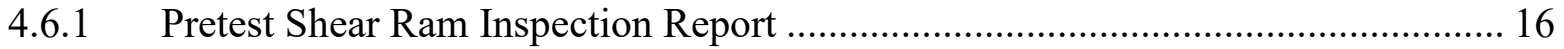

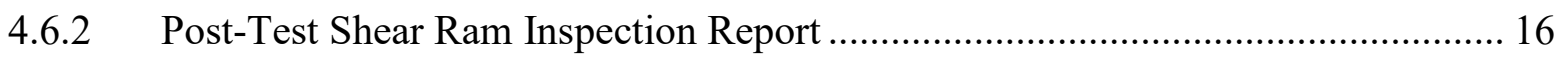

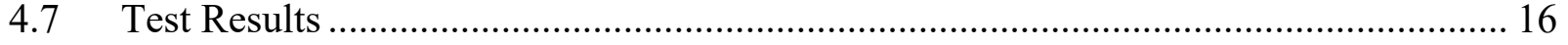

4.8 Photographic and/or Video Records ...................................................................... 18

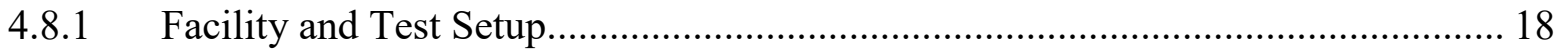

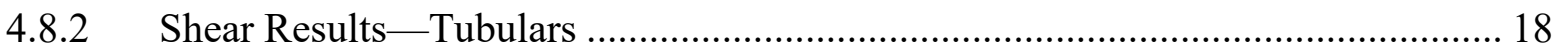

4.8.3 Shear Ram Condition (Before and After) Tests.................................................... 19

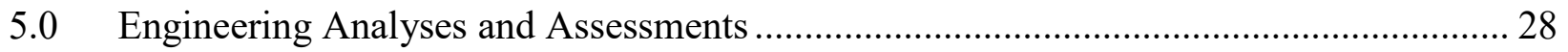

$5.1 \quad$ Theoretical Shear Calculations................................................................................... 28 


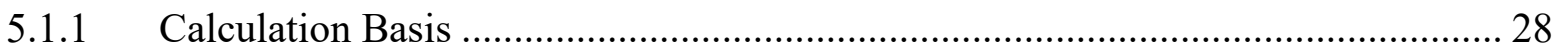

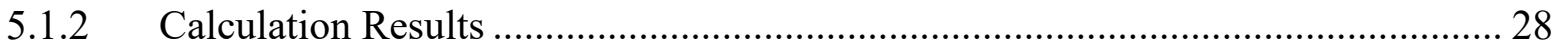

5.1.3 Compare Calculations to Test Results ............................................................. 29

5.2 Shear Ram Support Systems Capability Assessment...................................................... 29

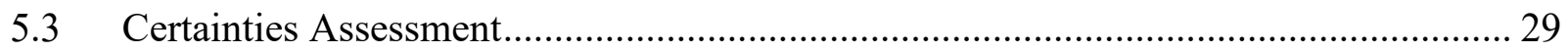

5.3.1 Expected Variation in Test and Calculated Shear Pressures ................................. 29

5.3.2 Comparisons of Rig Versus Tested Equipment ................................................... 30

5.3.3 Available Maximum Hydraulic Pressure for Adjusted Peak Shear Pressure ......... 31

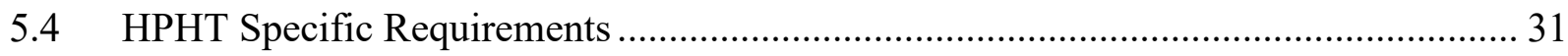

6.0 Statement on Shear Ram and System Fitness for Service ………………....................... 32

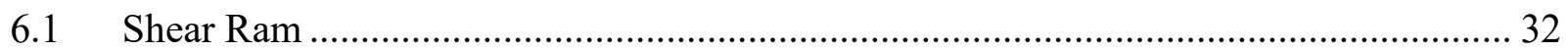

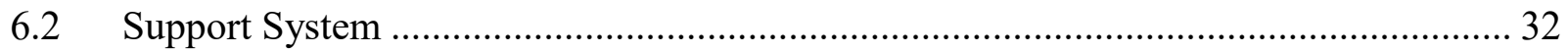

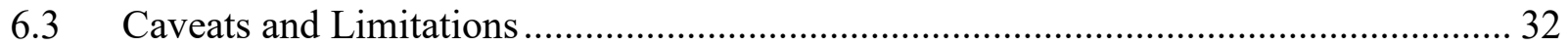

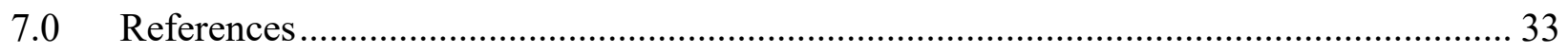

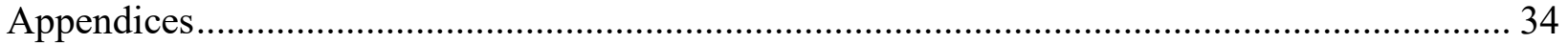

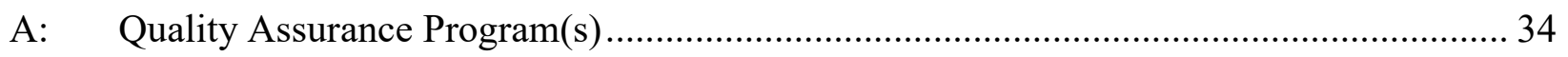

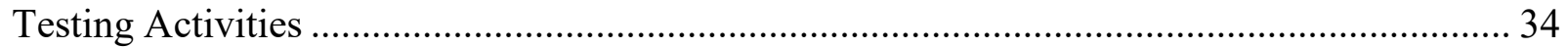

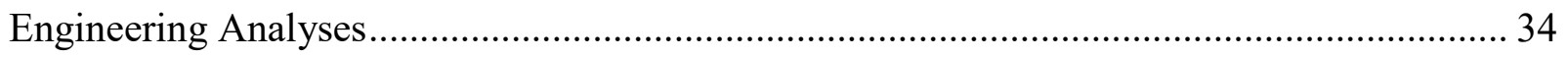

B: $\quad$ Personnel Qualification and Credential Records .............................................................. 35

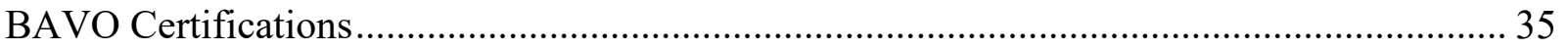

Professional and Engineering Qualifications/Registrations ........................................................ 35

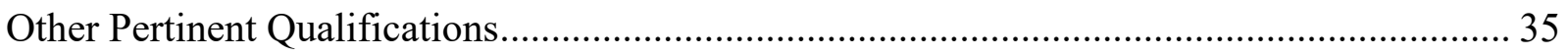

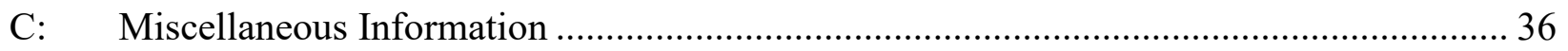

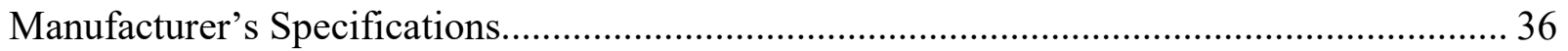

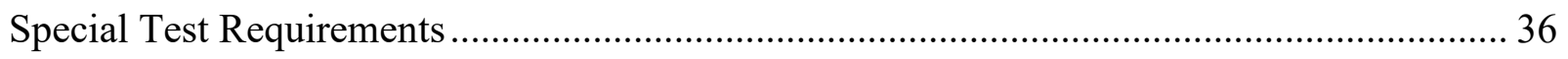

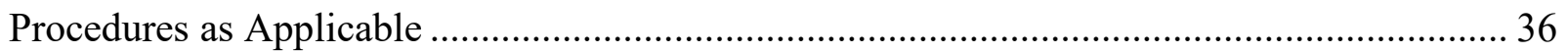

Tubular or Wire Chemistry .......................................................................................... 36

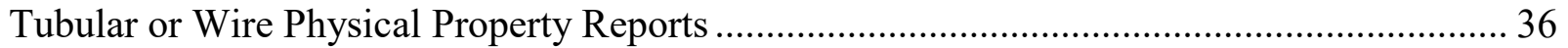

Pre- and Post-Test Inspection Reports ............................................................................... 36

Raw Shear Test Data or Recording Charts........................................................................ 36 


\section{LIST OF TABLES}

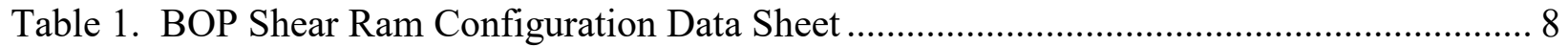

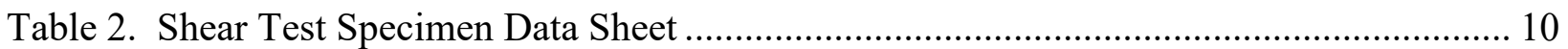

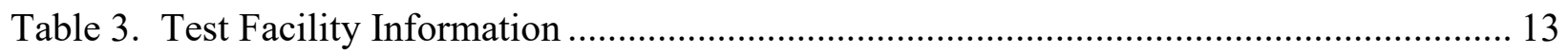

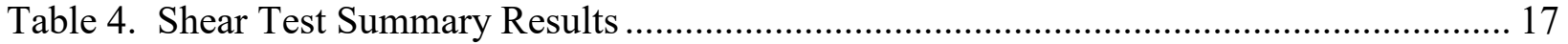

\section{LIST OF FIGURES}

Figure 1 Placeholder for Shear Ram Test Support Systems at (Name) Facility (Images, Figures, and/or Drawings of control, data acquisition, and mechanical Equipment) ............................... 20

Figure 2 Example Photographs of Shear Ram Ready for Shear Testing ..................................... 21 Figure 3 Placeholder for Tubular Specimen and Specimen Support Apparatus (Images, Figures,

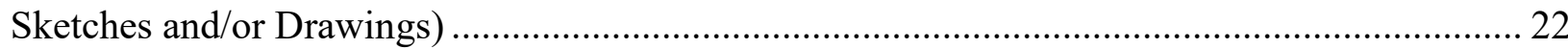

Figure 4 Placeholder for Shear Specimen in Bore of shear Ram Just Prior to Shear Test (Images) (n)

Figure 5 Placeholder for Ram Blocks Before and After Shear and Seal Test(s) (Images).......... 24 Figure 6 Example Photograph of Sheared Tubular (Identification) Showing Shape Distortion

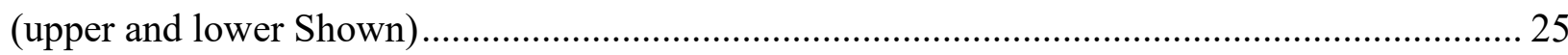

Figure 7 Placeholder for Shear Ram Seal Assembly (Before and After Seal Testing-Images) .. 25

Figure 8 Placeholder for Shear Ram Inspection Underway (Images/Diagrams)......................... 27 


\subsection{Introduction}

The Bureau of Safety and Environmental Enforcement (BSEE) Well Control Rule (Subpart G) ${ }^{1}$ was published on April 29, 2016, and became effective on July 28, 2016. This rule is part of BSEE regulations pertaining to well control equipment used for oil and gas operations on the U.S. Outer Continental Shelf (OCS).

In accordance with this new rule, BSEE seeks to obtain specific information from operators regarding the capabilities of shear rams in the Blowout Preventer (BOP). This information is required prior to beginning any operation involving the use of any BOP. Title $30 \mathrm{CFR}$ 250.732(c)(2) of these regulations requires documentation of tests and associated engineering analyses and calculations to demonstrate the shearing and sealing capabilities of the BOP. Such documentation is consistent with and complementary to other documentation verifying the designs of individual components and the overall BOP system. Such verification is part of a testing process that demonstrates the performance and reliability of the equipment in a manner that is repeatable and reproducible. ${ }^{2}$

This document provides guidance concerning how to report BOP shear ram tests to support BSEE's regulatory role. The following information is suggested content:

1. Design and operational details about the specific shear ram(s) tested

2. Technical details on any tubulars and wirelines sheared

3. Description of the testing facility, test equipment, test equipment calibration, and certification

4. Testing procedures and methods used

5. Test results and acceptance criteria for the shearing tests and sealing tests

6. Design and operational details about the specific shear ram(s) for well control operations on the actual rig

7. Technical details on all tubulars and wirelines on the actual rig

8. Engineering calculations and analyses related to the testing and reconciliation of any technical differences between the testing and the actual intended operation

9. The quality assurance program(s) applied to the verification process

Moreover, a BSEE-approved verification organization $\left(\mathrm{BAVO}^{3}\right)$ is to be used for certification, verification, and reporting of the BOP shear ram tests. (Please note that the BAVO requirements addressed in this document pertain only to BOP shearing and sealing.)

\footnotetext{
${ }^{1}$ Appearing in BSEE regulations at Title 30, Chapter II, Subchapter B, Part 250, Subpart G "Well Operations and Equipment."

2 See Title 30 CFR $\$ 250.732$ (b)(1)(ii) “....use of test protocols and analysis that represent recognized engineering practices for ensuring the repeatability and reproducibility of the tests, and that the testing was performed by a facility that meets generally accepted quality assurance standards."

${ }^{3}$ See Title 30 CFR $§ 250.732$ : BAVO requirements. BSEE is engaged now in developing BAVO acceptance criteria. BAVO information in this document will be updated when appropriate.
} 
For high-pressure, high-temperature (HPHT) well conditions, there are additional reporting requirements. These requirements appear in a section of this document. In the event of a conflict between any regulation(s) and this document, the regulation(s) take precedence.

This document recommends a format and organization for the presentation of technical data. The actual submittal need not follow this template, but the content should be consistent with this document as shown beginning with Section 2.0. Conceptually, with documentation, the reported tests could be repeated with similar results without other input or knowledge. 


\subsection{General Information}

\section{$2.1 \quad$ Rig}

At a minimum, provide the unique name and ownership of the drilling rig that is the reference of the report.

\subsection{Well(s)}

Provide the name(s) and lease identification details associated with the well(s) for which the shear ram verification is prepared. This shear report may pertain to several wells, all of which may not be known for the initial submittal of the report. Supplementary documentation or an amendment to this section may associate the drilling rig, drill pipe, and blind shear ram(s) with additional well(s). However, the additional wells should fall within the capabilities envelope of the blind shear ram testing.

\subsection{HPHT Conditions}

State whether HPHT conditions ${ }^{4}$ are anticipated for the wells associated with the report. If they are, regulations require supplemental information and assessments not required for nonHPHT wells. See Section 5.4 for more details.

${ }^{4}$ See CFR Title 30, Volume 2 Section 250.804(b) for complete description. 


\subsection{Drilling Rig Equipment}

\subsection{Drilling Rig Shear Ram}

Table 1 is a dual-purpose table that captures information for the rig shear ram(s) (similar to the tested shear ram in Section 4.2). When the actual rig equipment is not tested, multiple tables need to capture comparable information to support post-test engineering assessments. The table identifies whether the shear ram is actual rig equipment or representative equipment used for shear ram performance testing. In addition, the table (and other similar tables) includes consistent quality assurance information, including:

- quality assurance representative(s);

- other responsible individuals;

- individual affiliations and titles/roles; and

- $\operatorname{date}(\mathrm{s})$.

\section{TABLE 1. BOP SHEAR RAM CONFIGURATION DATA SHEET}

\section{BOP and BOP System Equipment}

Identify whether information in this table pertains to actual rig equipment or representative equipment for the reported shear testing (check one).

BSR Equipment: $\square$ Actual rig BSR and actuator or $\square$ Representative rig BSR and actuator

\section{\begin{tabular}{|l|l|l}
\hline Blowout Preventer (Shear Ram & Value and Units & Comments and Notes
\end{tabular}}

\section{Only)}

\begin{tabular}{|l|l|l|}
\hline Manufacturer & & \\
\hline Description and Type & & \\
\hline Bore Size & & \\
\hline BOP Serial Number (s) & & \\
\hline Housing Manufacture Date & & \\
\hline Wellbore Rated Working Pressure & & \\
\hline $\begin{array}{l}\text { Wellbore Rated Working } \\
\text { Temperature }\end{array}$ & & \\
\hline $\begin{array}{l}\text { Refurbishment and Inspection } \\
\text { History }\end{array}$ & & Comments and Notes \\
\hline Blind Shear Ram & Value and Units & \\
\hline Ram Manufacturer & & \\
\hline Description and Type & & \\
\hline
\end{tabular}




\begin{tabular}{|c|c|c|}
\hline Centering Feature $^{1}$ & & \\
\hline Ram Block Part Number(s) & & \\
\hline Ram Serial Number(s) & & \\
\hline Date of Manufacture & & \\
\hline Seal Manufacturer & & \\
\hline Seal Part Number & & \\
\hline Seal Durometer @ & & \\
\hline BOP Blind Shear Ram Actuator & Value and Units & Comments and Notes \\
\hline Manufacturer & & \\
\hline Description and Type & & \\
\hline Main Assembly Number(s) & & \\
\hline Serial Number(s) & & \\
\hline Date of Manufacture & & \\
\hline $\begin{array}{l}\text { Rated Working Pressure (Main } \\
\text { Actuator(s)) }\end{array}$ & & \\
\hline $\begin{array}{l}\text { Other Nameplate Information (e.g., } \\
\text { Part Numbers, API Std, etc.) }\end{array}$ & & \\
\hline $\begin{array}{l}\text { Manufacturer's Maximum } \\
\text { Specified Shearing Pressure }\end{array}$ & & \\
\hline Piston Closing Area & & \\
\hline Piston Opening Area & & \\
\hline Piston Rod Area & & \\
\hline Piston Rod Stroke & & \\
\hline Closing Ratio (Pressure Ratio) & & \\
\hline $\begin{array}{l}\text { Quality Assurance Record } \\
\text { Information }\end{array}$ & & \\
\hline $\begin{array}{l}\text { Preparer, Concurrence Name, Date, } \\
\text { Affiliation, etc. }\end{array}$ & & \\
\hline
\end{tabular}

\subsection{Drilling Rig Shearable Tubulars and Wireline}

Similar to Table 1, Table 2 captures information about tubulars and wirelines used for the wells identified in Section 2.2. Conceptually, the table would be prepared for each type of drill pipe on the rig identified in Section 2.1. The example table may not be suitable to communicate all information. For example, materials chemistry, quality inspections or certification records, and physical properties would need to be appended for completeness. To minimize confusion, a clearly identified single table is recommended for each tubular or wireline. 


\section{TABLE 2. SheAR Test SPECIMEN DATA SheET}

\section{Test Specimen, Workstring or Tubing}

Identify whether the information in this table represents actual rig tubulars/wirelines or representative tubulars/wirelines (check box below). Provide a shear test specimen data sheet for any electric-, wire-, and slick-line sheared (information for each size and type of drillpipe, workstring, and tubing to be used in the well and not sheared to verify performance of shearing per 30CFR 250.732). For items not sheared in tests, similar information should be provided with calculations and engineering analyses.

Type of Tubular or Line: $\square$ Actual rig sample or $\square$ Representative sample

\begin{tabular}{|l|l|l|}
\hline Description & Value and Units & $\begin{array}{l}\text { Spec. Value and Units, } \\
\text { Comments and Notes }\end{array}$ \\
\hline $\begin{array}{l}\text { Tubular Type (e.g., Drill Pipe or } \\
\text { Wireline) }\end{array}$ & & \\
\hline Manufacturer & & \\
\hline Grade or Specification & & \\
\hline Nominal OD (in) & & \\
\hline Nominal ID (in) & & \\
\hline Weight per ft. & & Spec. Value and Units, \\
\hline Date of Manufacture & & \\
\hline $\begin{array}{l}\text { Source (Specify Rig Name or } \\
\text { Other) }\end{array}$ & & \\
\hline $\begin{array}{l}\text { Physical Properties (as Specified } \\
\text { in API 5DP) }\end{array}$ & Value and Units & \\
\hline Yield Strength (ksi) & & \\
\hline Tensile Strength (ksi) & & \\
\hline Elongation (\%) & & Spec. Value and Units, \\
\hline Reduction of Area (\%) & & \\
\hline Charpy (ft-lb at F) & & \\
\hline Hardness (HRC) and Location & & \\
\hline Chemical Properties & & \\
\hline Chemistry & & \\
\hline Pipe Material Test Reports & & \\
\hline Ouffiliation, etc. & & \\
\hline Information & & \\
\hline
\end{tabular}




\subsection{Drilling Rig Shear Ram Support Systems}

Rig support systems for the blind shear ram include hydraulic supply pressure at the entry to the ram, the type of fluid required, the total volume required to close, and flow rates. The engineering systems analysis should be consistent with this information, and shear testing should represent anticipated field conditions.

\subsection{Anticipated Well Conditions Pertinent to Shear Ram}

\subsubsection{Well Parameters}

Anticipated well environmental conditions and ranges of conditions that are important to shear ram performance should be reported. These include, but are not limited to:

- location;

- water depth;

- total vertical depth;

- total well depth; and

- chemistry (hydrocarbons, drilling fluid, acids, H2S, etc.).

\subsubsection{Maximum Anticipated Surface Pressure (MASP)}

The regulations at 30 CFR 250.734(a)(1)(ii) require that at least one shear ram be capable of sealing the wellbore after shearing under maximum anticipated surface pressure (MASP) ${ }^{5}$ conditions as defined for the operation. Per 30 CFR 250.730(a), the working-pressure rating of each BOP component (excluding annular(s)) must exceed MASP as defined for the operation. For a surface BOP, MASP is to be calculated at the surface. For a seafloor BOP, MASP is calculated at mudline conditions.

Adjustment of shear pressure for MASP is mandatory for appropriate engineering comparisons of shearing requirements to hydraulic supply capabilities. Reported shearing pressure requirements with MASP adjustment must never exceed the rig's available hydraulic supply pressure (e.g., accumulator) or rated working pressure. ${ }^{6}$

\footnotetext{
${ }^{5}$ MASP is defined in CFR $\$ 250.448(b)$.

${ }^{6}$ API Standard 53 ( ${ }^{\text {th }}$ ed. November 2012) at 6.5.10.7.5 : "Due to the variations in pipe properties and corresponding shear pressures, the maximum expected pressure for shearing pipe should be less than $90 \%$ of the maximum operating pressure. An additional risk assessment should be performed if the shear pressure is higher than $90 \%$ of the maximum operating pressure."
} 


\subsubsection{Maximum Anticipated Well Pressures and Temperatures}

Shear ram performance documentation includes maximum anticipated well pressures and temperatures. If these exceed HPHT thresholds, additional information is required per the regulations (see Section 5.4.) 


\subsection{Shear Ram Testing Information}

\subsection{Testing Facility Details}

Document facts and details about the testing facility, as listed in Table 3 (or equivalent).

TABLE 3. TEST FACILITY INFORMATION

\begin{tabular}{|c|c|}
\hline Test racility & $\begin{array}{l}\text { Comments/Notes (Aftach Addifitional Pages as } \\
\text { Needed) }\end{array}$ \\
\hline General Information Required & Enter Information. \\
\hline \multicolumn{2}{|l|}{ Facility Name } \\
\hline \multicolumn{2}{|l|}{ Facility Physical Address } \\
\hline \multicolumn{2}{|l|}{ Test Supervisor Name and Title } \\
\hline \multicolumn{2}{|l|}{ Client Name and Address } \\
\hline \multicolumn{2}{|l|}{$\begin{array}{l}\text { Client's Technical POC Name, Title, } \\
\text { and Contact Information }\end{array}$} \\
\hline \multicolumn{2}{|l|}{$\begin{array}{l}\text { Witnesses (e.g., Third Party, BAVO, } \\
\text { Client, etc.) Name, Title and Contact } \\
\text { Information }\end{array}$} \\
\hline Hydraulic System (for Example) & Enter Imformation. \\
\hline \multicolumn{2}{|l|}{$\begin{array}{l}\text { Pump Nameplate Make, Model, Size, } \\
\text { HP, Rating. S/N, etc. }\end{array}$} \\
\hline \multicolumn{2}{|l|}{$\begin{array}{l}\text { Regulator Make, Model, Size, S/N, } \\
\text { Test Setting (psig), etc. }\end{array}$} \\
\hline \multicolumn{2}{|l|}{$\begin{array}{l}\text { Maximum Manufacturer-Rated } \\
\text { Working Pressure (psig) }\end{array}$} \\
\hline \multicolumn{2}{|l|}{ Maximum Rig Supply Pressure (psig) } \\
\hline \multicolumn{2}{|l|}{ System Configuration (Attach P\&D) } \\
\hline \multicolumn{2}{|l|}{$\begin{array}{l}\text { Test Hydraulic Fluid Product Trade } \\
\text { Name and Mixture }\end{array}$} \\
\hline Data Acquisition System ${ }^{1}$ & Define and Characterize System. \\
\hline \multicolumn{2}{|l|}{$\begin{array}{l}\text { Chart Recorders - Digital Acquisition } \\
\text { System Manufacturer(s), Model(s), } \\
\text { S/N, Calibrations and Validations }\end{array}$} \\
\hline Instrumentation $^{1}$ & Define and Characterize System \\
\hline $\begin{array}{l}\text { Closing Pressure, Chamber Pressure } \\
\text { Transducer Manufacturer, Model, } \\
\text { S/N, etc. }\end{array}$ & $\begin{array}{l}\text { E.g., Maximum Scale (25-75\% of Full Scale), } \\
\text { Accuracy ( } \% \text { of Full Scale) Records of Last } \\
\text { Calibration, Measurement Frequency, etc. }\end{array}$ \\
\hline \multicolumn{2}{|l|}{$\begin{array}{l}\text { Opening Pressure, Chamber Pressure, } \\
\text { Transducer, and Location on BOP }\end{array}$} \\
\hline \multicolumn{2}{|l|}{ Flowmeters } \\
\hline Backup or Secondary P-Transcucers & \\
\hline
\end{tabular}




\begin{tabular}{|l|l|}
\hline Measuring and Examination & \\
\hline Quality System & Indicate Whether Measured or Specification. \\
\hline Standard & Identify Standard, System Implemented, etc. \\
\hline Last Audit (MDY) & $\begin{array}{l}\text { Audit Findings and Certification Period } \\
\text { Coverage }\end{array}$ \\
\hline $\begin{array}{l}\text { Quality Assurance Record } \\
\text { Information }\end{array}$ & \\
\hline $\begin{array}{l}\text { Preparer, Concurrence Name, Date, } \\
\text { Affiliation, etc. }\end{array}$ & \\
\hline
\end{tabular}

\subsection{Shear Ram(s) Tested}

Shear ram performance documentation must provide design and operational details about the specific shear ram(s) tested. If the equipment tested is not the actual rig shear ram (or one of the rig's identical shear rams), Table 1 (above) has a dual purpose and can be used to capture the information for either piece of equipment (tested or rig shear rams). When the tested equipment is not the actual rig equipment, the table contains comparable information that supports post-test engineering performance assessments.

${ }^{1}$ Blind shear descriptions need to include the pipe-centering device, and that device should be part of the blind shear testing (30 CFR 250.734 (a)(16)). Per Subpart G, the centering device must be installed in the BOP no later than May 1, 2023.

BOP stack traceability shall include information about the quality system applied by BOP manufacturer(s). This includes QA certification by an entity meeting the requirements of $30 \mathrm{CFR}$ $250.730(d)$.

\subsection{Test Setup}

The testing documentation must present details of the actual shear ram placement in the facilities test setup. Important topics include the following:

- Interfaces with facility test system (hydraulic connections, instrumentation, etc.)

- Shear ram sensors and transducers and locations (and current calibration certificates)

- Test specimen support system hardware

- Test specimen constraints (mechanical apparatus features)

- Centering mechanism (if any)

- Leakage examination (procedure and acceptance criteria)

- Assembly and makeup of the shear ram assembly (photograph, manufacture requirements) 
- Piping and instrumentation diagram (P\&ID)

- Position of test specimen in bore

These subjects must be consistent with the test procedure.

\subsection{Test Procedure/Protocol Followed and Acceptance Criteria}

The procedure/protocol used for the shear ram must be documented. All steps of the test sequences, including any exceptions, must be listed. Also, a sound protocol should include acceptance criteria. Independent of protocol source or reference documentation, this must be a step-by-step description of test procedures, beginning with sample preparation and proceeding through ram block and operator function tests, pipe sample positioning, pipe shearing and sealing tests, and inspections.

Justification for off-center and side-load positioning and shearing of pipe must be informed. ${ }^{7}$ This is to be consistent with regulatory implementation schedules (see the individual sections of 30 CFR 250.700+ for effective dates of the Well Control Rule subsections).

Specific criteria and subjects are a component of documentation about testing that adequately supports BAVO organization verification roles (see 30 CFR 250.732). Examples of these areas of BAVO verification include the following:

- Test equipment

- Facility and personnel qualifications

- Representation of test facility relative to actual rig and field conditions

- Shear and seal pressure and temperatures

- Data recording/capturing and reporting of test results

\subsection{Test Specimen Selection}

This section must inform how shear test samples were chosen and prepared and how the samples are typical of the rig tubulars (drill pipe, workstring, tubing, electric lines, wirelines, etc.) to be used in the well. This should address metallurgy, design specifications/standards, actual physical and chemical properties, size variations, sample preparation cutting methods, length of test

\footnotetext{
${ }^{7}$ Conceivably these could be somewhat design dependent with regard to the most challenging shearing situation in terms of shearing force or post shear sealing. For such a circumstance, prudent engineering practice and risk management would address these distinctions.
} 
sample, and position in test specimen section. For hot cutting, a heat-affected zone is shown outside the shear test plane on the test specimen and includes technical assurances regarding the extent of any heat affected zones.

\subsection{Shear Ram Inspection Information}

\subsubsection{Pretest Shear Ram Inspection Report}

Prior to testing a shear ram, there should be full physical inspection documentation as to the condition.

The scope of the inspection includes the following:

- Shear blades

- Ram blocks

- Sliding surfaces of the ram block

- Centering mechanism (as applicable)

- Seal surfaces

- Elastomers and seals

- Housing,

- Operator(s)

This documentation identifies the applicable manufacturer's specifications and indicates whether the shear ram assembly complies. If modifications occur during testing, the nature and extent of such should also be described. Finally, include BAVO verification of tested BOP shear ram assembly condition (third party prior to BAVO implementation).

\subsubsection{Post-Test Shear Ram Inspection Report}

As outlined in the paragraph 4.6.1, a post-test inspection is to be reported as part of the verification of general shear ram performance. This would include a qualified statement that the unit complies with applicable manufacturer's specifications and is suitable for subsea service (with possible parts replacements) as intended for the well conditions described in Section 2.2.

\subsection{Test Results}

Table 4 is a suggested format to summarize the results of each shear test. Actual recorded data should accompany such charts. This data could be plotted digital data or recorder charts with 
notations of key events and visual documentation (photographs or video--see Section 4.8 for further details), including the following:

- BOP shear ram speed and pressures throughout the test (e.g., circular charts or other visual documentation)

- Start/end of test

- Initial specimen-ram blade contact, and point of shear ${ }^{\mathbf{1}}$

- Start/end of block travel

- End of test

- Indication of whether peak pressure exceeded manufacturer's specification or limitation of the BSR component

- Pressure test start

- Pressure buildup

- Hold pressure hold

- Pressure reduction

- End pressure test

- Operator hydraulic closing and opening areas

- NDE examination results (before, after, and as applicable during testing series)

\section{TABLE 4. SHEAR Test Summary Results}

\section{BOP Shear Test Results}

Identify whether the information in this table represents actual rig tubulars/lines or representative tubulars/lines (check one item per line below).

Test Sample Orientation : $\square$ Centered $\square$ Off Center (unsecured) or $\square$ Fixed To Wall (secured) or $\square$ Other (explain)

Test Sample No. (if more than 1): $\square$ One $\square$ Two $\square$ Three or $\square$ Other (No. $\_$)

\begin{tabular}{|l|l|l|}
\hline Test Data Summary & Value and Units & Comments/Notes \\
\hline $\begin{array}{l}\text { Date of Shear (DMY and } \\
\text { Time of Shear) }\end{array}$ & $\begin{array}{l}\text { Success } \\
\text { or Failure: }\end{array}$ \\
\hline Opening Pressure (psi) & & \\
\hline Opening Volume (gal) & & \\
\hline Peak Close Pressure (psi) & & \\
\hline Peak Close Volume (gal) & & \\
\hline $\begin{array}{l}\text { Peak Shearing Force @ POS } \\
\text { (kips) }\end{array}$ & & \\
\hline
\end{tabular}




\begin{tabular}{|l|l|l|}
\hline $\begin{array}{l}\text { Sheared Specimen } \\
\text { Description/ID }\end{array}$ & & \\
\hline Specimen Measured OD (in) & & \\
\hline Low Pressure Test (psi) & & \\
\hline High Pressure Test (psi) & & \\
\hline Ram Closure Time (sec) & & \\
\hline Upper Specimen, H x W (in.) & & \\
\hline Lower Specimen, H x W (in.) & & \\
\hline Visual Examination ${ }^{1}$ & & \\
\hline $\begin{array}{l}\text { Quality Assurance Record } \\
\text { Information }\end{array}$ & & \\
\hline $\begin{array}{l}\text { Preparer, Concurrence Name, } \\
\text { Date, Affiliation, etc. }\end{array}$ & & \\
\hline
\end{tabular}

${ }^{1}$ Visual examination to include photos of test specimen, shear blades, ram packers, and other critical components prior to and after testing and be made part of the report.

\subsection{Photographic and/or Video Records ${ }^{8}$}

The general objective of this documentation should be to capture the testing from setup to posttest assessments and inspections.

\subsubsection{Facility and Test Setup}

Photography of the facility test system and test setup (Figure 1, Figure 2, Figure 3, and Figure 4 ) accompanies piping and instrumentation diagrams (P\&ID)s and includes details of the actual shear ram tested. Photographic documentation is marked to positively associate the information with other information in the shear ram performance assessment report (for example, the shear ram, client, date, and time).

\subsubsection{Shear Results-Tubulars}

${ }^{8}$ Note: the pictures and figures in this report do not have associated copyright release. 
The report contains photography of sheared specimens (as in Figure 5). This documentation should be marked for positive association with other tables, charts, and narrative in the shear report.

\subsubsection{Shear Ram Condition (Before and After) Tests}

Provide photographic documentation of the shear ram cutting and sealing mechanism before and after test shears (Figure 6 and Figure 7). Identify and characterize any repairs or alterations performed between tests. Logically this would include examinations verifying conformance with the manufacturer's specifications. 
This is visual documentation of the blind shear ram test setup including supporting systems and controls as detailed in Table 3. This may require several images, figures, and/or drawings. Part or all could be a video or video monitor screen shots. 
Figure 2 EXAMPLE PHOTOGRAPHS OF SHEAR RAM READY FOR SHEAR TESTING

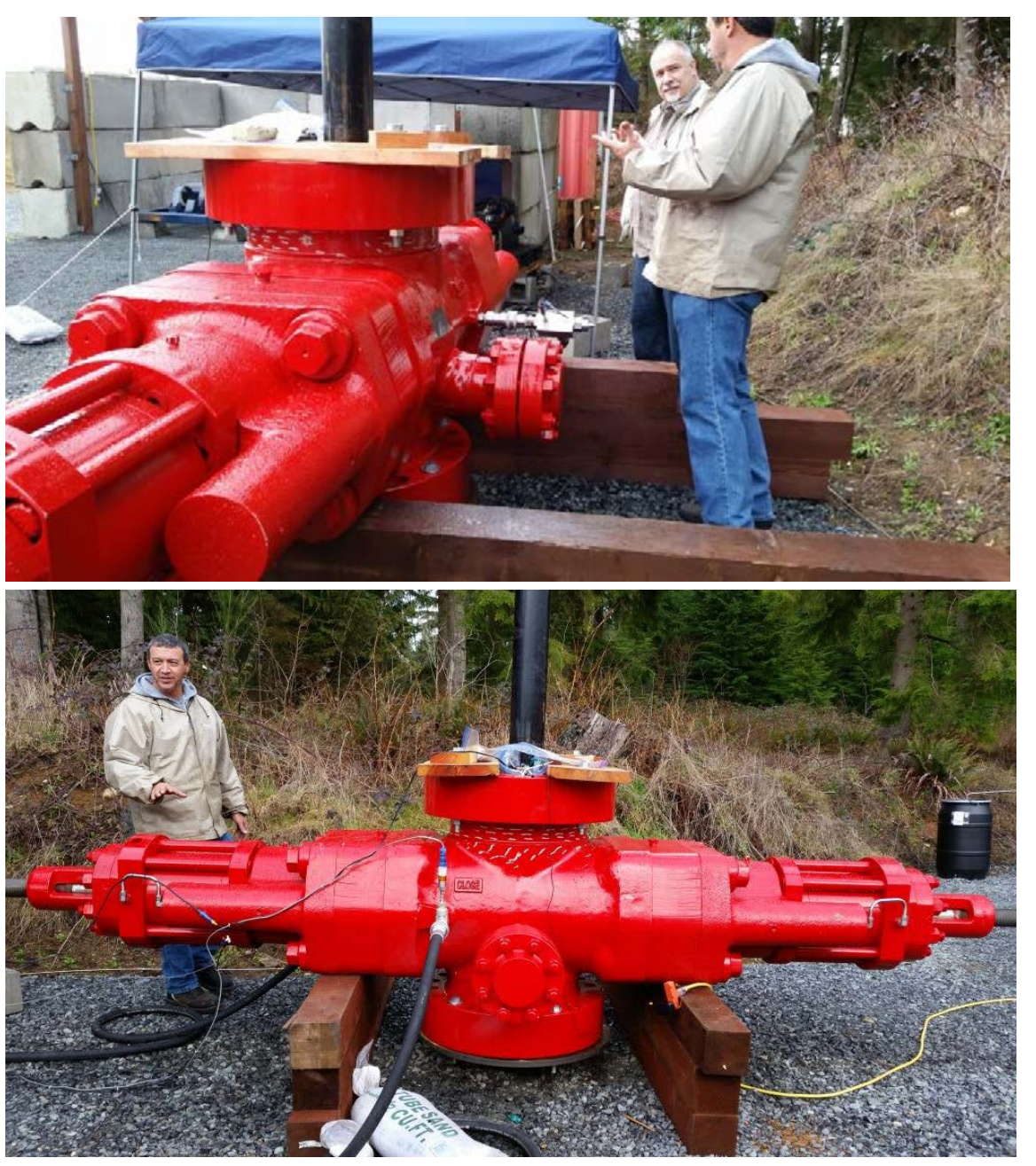

This is visual documentation of the BOP shear ram test setup prior to testing. This may require several images to document beyond the examples provided here. Part or all could be a video.

Above pictures reproduced courtesy of Bastion Technologies, Inc. 
Figure 3 Placeholder for Tubular Specimen and Specimen Support Apparatus (Images, Figures, Sketches and/or Drawings)

This is visual documentation of how the tubulars (listed in Table 2) are supported for the shear tests including any constraint hardware in the bore area. This may require several images, figures, sketches, and/or drawings. Part of documentation could be video. 
This is visual documentation of tubulars (listed in Table 2) in the well bore ready to be sheared. This may require several images to illustrate different tubulars and the test conditions defined in the regulations. Part of documentation could be videos. 
This is visual documentation of the ram blocks/shear blades before and after shear testing. Logically this includes information about shear cutting edge condition, restoration, or shear blade replacement during the test sequence. 
FiguRE 6 EXAMPLE PHOTOGRAPH OF SHEARED TUBULAR (IDENTIFICATION) SHOWING SHAPE DistorTiON (UPPER AND LOWER SHOWN)

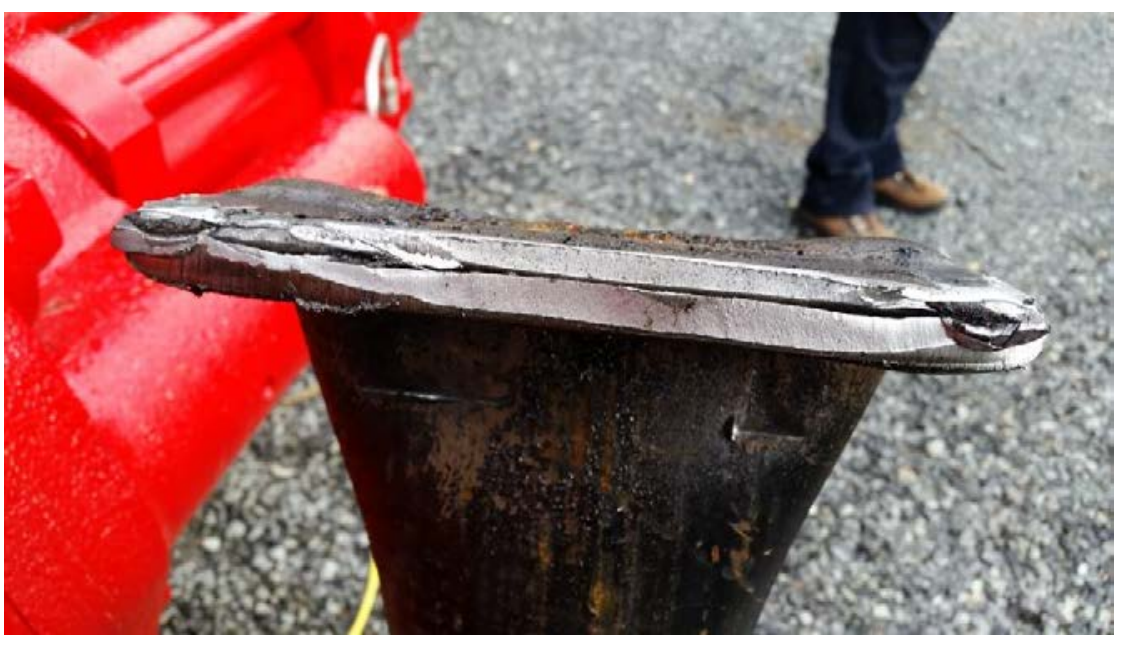

Reproduced courtesy of Bastion Technologies, Inc.

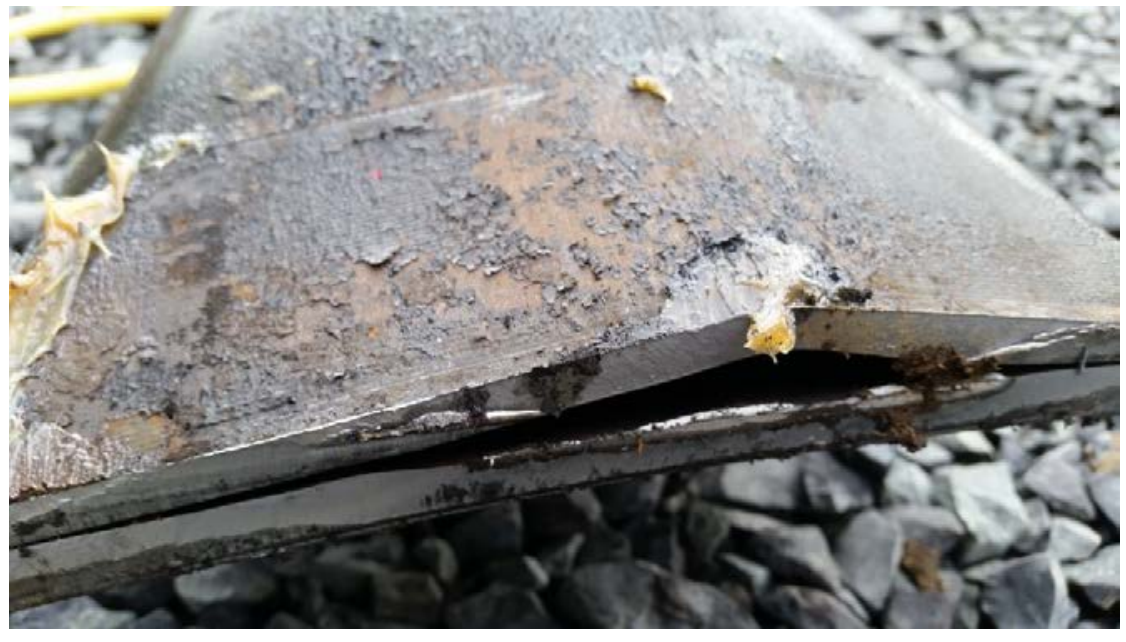

Reproduced courtesy of Bastion Technologies, Inc. 
Figure 7 Placeholder for Shear Ram Seal Assembly (Before and After SeAl Testing-Images)

This is visual documentation of the BOP shear ram seals - pre and post testing. 
Good practice is to verify that the shear ram assembly complies with manufacturer's specifications particularly before the test. Post-test verification is logical in the interest of safety independent of whether the tested assembly is placed into service after testing. 


\subsection{Engineering Analyses and Assessments}

Sections 2.0, 0, and 0 document information about the well, the drilling rig and rig support systems for BOP(s), the specific BOP(s) used by the drilling rig, the BOP shear ram actually used for shear tests, the rig drill pipe and wire, and shear test results. Through all this, the simplest case is when the rig's BOP is used for actual shear testing with tubular and wire samples from rig stock. In this instance, there are the fewest concerns regarding how representative test results are of actual subsea operations and field conditions. On the other extreme, a tested BOP shear ram could be markedly different from a rig BOP shear ram, as could the tubulars and wires actually sheared. For such situations, there are technical concerns and uncertainties regarding the predicted performance of the actual BOP. In addition to the normal adjustments associated with BOP testing in a controlled environment (as compared to a subsea one), a thorough engineering analysis would identify and quantify performance differences between the actual rig BOP and the tested equipment. The ultimate goal is to demonstrate that shearing can occur with certainty and that there is adequate design margin to do so. The following section 0 identifies key areas of such engineering comparisons and analyses.

\subsection{Theoretical Shear Calculations}

A comparison of current test results to historical experience can be useful to address issues such as whether the rig BOP support system has adequate supply pressure to shear all tubulars.

\subsubsection{Calculation Basis}

There are engineering methods and formulas for predicting shear force for a given tubular and shear ram combination. Some methods could arise from fundamentals, while others may be empirical (such as a correlation of data collection). Independent of the basis, documentation should provide formula(s), values for constants, definitions of variables and associated units, and narratives about whether a particular formula is specific to the shear blade designs of the situation and whether the formula results represent the average or some other portion of supporting data. Conceptually, a formula or mechanics model could even be purely theoretical, but some level of actual validation is necessary. The formula or predictive method(s) must be referenced sufficiently to enable BSEE personnel (based on the reference) to request and inspect the validation information and protect intellectual property.

\subsubsection{Calculation Results}


Normal documentation includes shear prediction calculations for the actual and sheared (if different from actual) tubulars. These need to be representative of the tubular and shear blade/actuator design combination tested. Complete supporting documentation shows the formula, variables and units, constant values, calculated results, and notes about whether the prediction pertains to actual rig equipment or tested equipment. A thorough presentation links the calculated results to the summary tables presented previously (or equivalent).

\subsubsection{Compare Calculations to Test Results}

Shear force calculations are one means to interpret shear test results. If a correlation is intended to capture 95 percent of or all data, yet the actual shear results are greater than the correlation, there would be considerable cause for concern, and an explanation would be necessary. If the test results fall substantially below a correlation producing an average force estimate, there is a lesser concern. Nevertheless, a technical/statistical explanation is still appropriate to quantify possible variances in the shear test results and the associated shear testing acceptance criteria adopted for the testing.

\subsection{Shear Ram Support Systems Capability Assessment}

The hydraulic system for shear testing should resemble field conditions and capabilities, but there may be differences out of practicality. Potential differences are the following:

- Hydraulic fluid type

- Supply pressures and pump flow rate at shear ram manifold

- Fluid temperature

- Regulatory set points,

- Different valves and line resistance

- Hydraulic head

- Volume of fluid available

The document should include an assessment of these and other differences regarding the performance of the actual subsea BOP shear ram. Presumably, the system should be able to power the shear actions based on the worst-case and most conservative shear requirements, whether they are calculated or actual test results.

\subsection{Certainties Assessment}

5.3.1 Expected Variation in Test and Calculated Shear Pressures 
Since any shear test can be unusually high or low and since possible variance is large when sample size is small, the relative certainty of test results is important. A statistically significant number of shear tests is unlikely to support shear performance because of the time and cost. The next best option is to compare calculations and data and know and show expected variances of that data. In so doing, conservative requirements can be determined for the shear ram support systems with a degree of certainty.

\subsubsection{Comparisons of Rig Versus Tested Equipment}

A shear and seal test performed at an onshore test facility (e.g., OEM test facility) cannot exactly duplicate in-field equipment, applications, and conditions (even if the rig's BOP and test specimen were used in the test at the onshore facility). Thus, a documented systems engineering assessment is necessary to evaluate substantial technical differences and similarities. These assessments must support the technical argument that the tests performed onshore reflect equipment, applications, and conditions in the field sufficiently to certify that the rig's shear rams, control system and components, and accumulator will shear all tubulars and lines in the hole. Some typical considerations are the following:

1. Adjustments to required shearing pressures (heads, mechanical losses, MASP, etc.)

2. Hydraulic fluid type and characteristics, including viscosity and thermal stability. Define and document technical specifications and capabilities of the entire hydraulic system supplying pressurized fluid to shear rams for shear testing (hydraulic fluid, pumps, valves, hoses, piping, regulator(s), etc.). Comparative assessments should include a risk assessment if shear pressure exceeds 90 percent of system capability. ${ }^{9}$

3. Physical, mechanical, and chemical pipe properties prior to shearing. ${ }^{10}$ In addition to the physical and mechanical properties (e.g., the wall thickness (in.), hardness (HRc), and outside diameter (in.)) identified in Table 2, which are required to be measured prior to shearing at the three locations along the pipe length, a placeholder line has been included for the pipe's chemical properties. Preferably, these chemical properties (as per pipe manufacturer) characterize significant variations between that from the pipe manufacturer and that measured prior to shearing as part of the sample preparation. This physical information can support technical explanations for certain variations or deviations.

4. Physical properties of sealing elastomers and temperature variations. The shear and seal tests may be conducted at $70^{\circ} \mathrm{F}$, but actual conditions could vary from near freezing to something considerably higher. Further, there are possible issues of chemical compatibility for a sealing elastomer with the anticipated fluids that may be present.

\footnotetext{
${ }^{9}$ See API Standard 53 (November 2012), at Sections 6.5.10.7.5 and 7.6.11.7.5 for details.

${ }^{10} 30$ CFR $\$ 250.732(b)(1)(v)$
} 


\subsubsection{Available Maximum Hydraulic Pressure for Adjusted Peak Shear Pressure}

A fundamental issue is whether the rig or field hydraulic system has adequate pressure capability to shear the rig tubulars and wires. While other limits may exist, one comparison should be the available supply pressure at the shear ram operator input based on the maximum hydraulic pressure required during the shear process. Normally, this is greater than the pressure required at the time of shear.

\section{$5.4 \quad$ HPHT Specific Requirements}

The Verification Test Report (this document, including clearly referenced supplements or addenda) shall contain additional information if the BOP stack is expected to experience HPHT environment. Certain verifications must be documented and submitted before beginning HPHT operations. ${ }^{11}$ The HPHT requirements include the following:

- Certification that the third-party verification organization conducted a detailed review of the BOP package design to ensure that all critical components and systems meet recognized engineering practices

- Verification that the designs of individual components and the overall system have been proven in a testing process that demonstrates the performance and reliability of the equipment in a manner that is repeatable and reproducible ${ }^{12}$

- Verification that the BOP equipment will perform as designed in the temperature, pressure, and environment that will be encountered

- Verification that the fabrication, manufacture, and assembly of individual components and the overall system uses recognized engineering practices and quality control and assurance mechanisms

- Complete material and quality control over contractors, subcontractors, distributors, and supplies at every stage in the fabrication, manufacture, and assembly process ${ }^{13}$

\footnotetext{
${ }^{11}$ See 30 CFR $\$ 250.732$ (c)

${ }^{12}$ Identification of all reasonable failure modes and evaluation of design verification tests. See CFR $\$ 250.732$ (c)(2)(i) and (ii) for complete regulatory language

${ }^{13} 30$ CFR $\$ 250.732(\mathrm{c})(4)$
} 


\subsection{Statement on Shear Ram and System Fitness for Service}

This section is a consolidated summary and conclusion from the testing and engineering analyses conducted for the proposed well drilling. This section clearly identifies the $\mathrm{BAVO}^{14}$ (once the requirement goes into effect), per 30 CFR 250.732.

\subsection{Shear Ram}

Specifically state and attest that the rig shear ram performance, as configured in the BOP system on the actual rig, is adequate to shear any and all rig tubulars and wire.

\subsection{Support System}

Specifically state and attest that the BOP support system on the rig is adequate to assure required performance of the subsea shear ram. Local supply pressure is a key consideration, but other technical issues should also be addressed if there is an expected limitation or constraint.

\subsection{Caveats and Limitations}

List any and all caveats and limitations associated with the statements on shear ram performance, associated support systems, and overall fitness for service. This would include any operational or compatibility areas found in the assessments (based on testing and engineering considerations) to perform at the proposed location, including the maximum environmental and operational conditions that the MODU is designed to withstand, as explained in 30 CFR 250.713 and 30 CFR 250.734.

\footnotetext{
${ }^{14}$ See Title 30 CFR $\$ 250.732$ BAVO requirements. BSEE is engaged now in developing BAVO acceptance criteria. BAVO information in this document will be updated when appropriate.
} 


\subsection{References}

List all references cited in the report. The information should be complete enough that BSEE can acquire the information from open sources or, if there are proprietary issues, request the information from sources not generally available to the public. 


\section{Appendices}

\section{A: Quality Assurance Program(s)}

\section{Testing Activities}

Provide summary of QA program or reference program documentation available to BSEE upon requests and explain applicability relative to testing facility. Topics include the following:

- Facility test equipment

- Data recording equipment

- Procedures

- Protocol process

- Audits

- Responsible personnel

- Quality documentation

Use API Spec Q1, $8^{\text {th }}$ edition equivalent as a minimum (officially incorporated by reference at 30 CFR 250.198) or later edition, based on justification per regulations. Also, complete documentation is expected to identify BAVO principals performing, reviewing, and approving the report content, along with their organizational affiliation and dates.

\section{Engineering Analyses}

Provide summary of QA program or reference program documentation available to BSEE upon requests and explain applicability relative to testing facility. Topics include the following:

- Procedures

- Peer reviews

- External (third party or BAVO) review

- Audits

- Responsible personnel

- Quality documentation

Use API Spec Q1, $8^{\text {th }}$ edition equivalent as a minimum or a later edition if officially incorporated by reference at 30 CFR 250.198). Also document the BAVO principals performing, reviewing, and approving the report content along with their organizational affiliation and dates. 


\section{B: Personnel Qualification and Credential Records}

BAVO Certifications

Professional and Engineering Qualifications/Registrations

Other Pertinent Qualifications 


\title{
C: Miscellaneous Information
}

\author{
Manufacturer's Specifications
}

Special Test Requirements

Procedures as Applicable

Tubular or Wire Chemistry

Tubular or Wire Physical Property Reports

Pre- and Post-Test Inspection Reports

Raw Shear Test Data or Recording Charts 


\section{Argonne}

\section{Energy Systems Division}

Argonne National Laboratory

9700 South Cass Avenue, Bldg. *\#362

Argonne, IL 60439

www.anl.gov

Argonne National Laboratory is a U.S. Department of Energy laboatory managed by UChicago Argonne, LLC 